\title{
1 Patient stratification reveals the molecular basis of disease
}

\section{2 comorbidities}

3 Beatriz Urda-García ${ }^{1,2}$, Jon Sánchez-Valle ${ }^{1, *}$, Rosalba Lepore $^{1,3}$ and Alfonso Valencia ${ }^{1,4, *}$

$4 \quad{ }^{1}$ Barcelona Supercomputing Center (BSC), Barcelona, 08034

$5 \quad{ }^{2}$ Department of Experimental and Health Sciences, Universitat Pompeu Fabra, Barcelona, Spain

$6{ }^{3}$ Department of Biomedicine, Basel University Hospital and University of Basel, Basel, Switzerland

$7 \quad{ }^{4}$ ICREA, Barcelona, 08010 Spain.

$8 *$ Correspondence to: jon.sanchez@bsc.es and alfonso.valencia@bsc.es

\section{Abstract}

Epidemiological evidence shows that some diseases tend to co-occur; more exactly, certain groups of patients with a given disease are at a higher risk of developing a specific secondary condition. Despite the considerable interest, only a small number of connections between comorbidities and molecular processes have been identified.

14 Here we develop a new approach to generate a disease network that uses the accumulating RNA-seq data on human diseases to significantly match a large number of known comorbidities, providing plausible biological models for such co-occurrences. Furthermore, $64 \%$ of the known disease pairs can be explained by analysing groups of patients with similar expression profiles, highlighting the importance of patient stratification in the study of comorbidities.

These results solidly support the existence of molecular mechanisms behind many of the known comorbidities. All the information can be explored on a large scale and in detail at http://disease- 
medRxiv preprint doi: https://doi.org/10.1101/2021.07.22.21260979; this version posted July 24,2021 . The copyright holder for this preprint (which was not certified by peer review) is the author/funder, who has granted medRxiv a license to display the preprint in perpetuity.

All rights reserved. No reuse allowed without permission.

\section{Introduction}

26 Disease comorbidity is defined as the co-occurrence of two or more conditions in the same patient ${ }^{1}$.

27 Comorbidity incidence increases with age and has a high impact on life expectancy, as it increases patient mortality and complicates the choice of therapies, posing a major problem for patients and health care systems. Accumulating evidence from epidemiological studies indicates that such co-occurrences do not appear randomly and that specific trends are observed, with some diseases co-occurring more than expected by chance ${ }^{2,3}$. Systematic studies using electronic health records have been performed to analyze comorbidity patterns in a given population where disease co-occurrences are represented by static networks ${ }^{2,3}$ or network trajectories if their progression over time is considered ${ }^{4}$. These studies demonstrated the predictive value of comorbidity patterns to determine disease progression and outcome, including mortality risk.

The observed patterns suggest that comorbid diseases might share underlying molecular mechanisms and risk factors, which can be both genetic and environmental, such as drug exposure and lifestyle. Thus, a better understanding of the molecular mechanisms behind comorbidities is a crucial step towards improved prevention, diagnosis, and treatment of these conditions.

Recent studies on disease comorbidities have included molecular information often analyzing pairs of diseases based on shared disease-related genes ${ }^{5}$. Similar to functionally related genes, diseaseassociated genes tend to colocalize in the protein-protein interaction network forming disease modules which can aid the identification of novel candidate genes and inform about disease associations, including phenotypic similarity and comorbidities ${ }^{6}$. In this context, previous work showed that the overlapping gene expression signatures between several Central Nervous System disorders (CNSd) and cancer types could inform about the molecular mechanisms underlying their direct and inverse comorbidities $^{7,8}$. More recently, we have observed that disease similarity networks based on gene expression profiles can be used to identify known comorbidity relationships ${ }^{8}$. Although these efforts were able to capture interesting examples, they were unable to recapitulate what is known at the medical level in a systematic manner. The mentioned approaches based on PPIs and microarrays reproduced a very small percentage of the epidemiology, and other networks based on miRNAs ${ }^{9}$ and the microbiome ${ }^{10}$ had no capacity for it. Hence, we address the still largely unknown extent to which molecular information can provide a general explanation to disease comorbidities.

54 Here, we reformulate the problem and we show, for the first time, that gene expression data is definitively able to reproduce medically known disease co-occurrences. To achieve that, we integrate 
medRxiv preprint doi: https://doi.org/10.1101/2021.07.22.21260979; this version posted July 24,2021 . The copyright holder for this preprint (which was not certified by peer review) is the author/funder, who has granted medRxiv a license to display the preprint in perpetuity.

All rights reserved. No reuse allowed without permission.

56

57

58

59

60

61

62

63

64

65

66

67

68

69

70

71

72

73

74

75

76

77

78

79

80

81

publicly available RNA-seq data sets, which are currently replacing microarrays due to their improved sensitivity, reproducibility, and detection's dynamic range ${ }^{11,12}$. We characterize the gene expression signature of human diseases based on differential expression and functional enrichment analyses. Then, we generate a disease similarity network based on the similarities between diseases' differential expression profiles. Afterwards, we build a stratified similarity network grouping patients of the same disease with a similar expression profile (here called meta-patients), addressing the fact that patients suffering from a given disease present different risks of developing specific secondary conditions, as evidenced by the Danish medical records ${ }^{4}$. Both networks are able to significantly recapitulate a large proportion (up to $64 \%$ ) of the medically known comorbidities ${ }^{2}$, providing a well-defined set of pathways and molecular functions potentially implicated in disease comorbidities, which can be analyzed at http://disease-perception.bsc.es/rgenexcom/.

\section{Results}

\section{Gene expression fingerprint of human diseases}

First, we collected published studies analyzing human diseases with RNA-seq data. Uniformly processed gene counts were obtained from the GREIN platform ${ }^{13}$. After quality filtering (see Methods), $58 \%$ of the samples were kept, corresponding to 2.705 samples from 62 studies and comprising 45 diseases (Supplementary Data 1).

We performed differential expression analyses to obtain significantly differentially expressed genes (sDEGs) for each disease (see Methods, Supplementary Table 1). As expected, the number of sDEGs positively correlates with the sample size, whereas it does not correlate with the average library size (average number of sequenced reads) of the diseases (Supplementary Fig. 1).

To better understand the transcriptomic alterations associated with the analyzed diseases, we performed functional enrichment analyses ${ }^{14}$ and, focusing on the significantly enriched Reactome pathways $(\mathrm{FDR}<=0.05)^{15}$, we clustered diseases based on their binarized Normalized Effect Sizes (see Methods, Fig. 1 and Supplementary Fig. 2-4). 


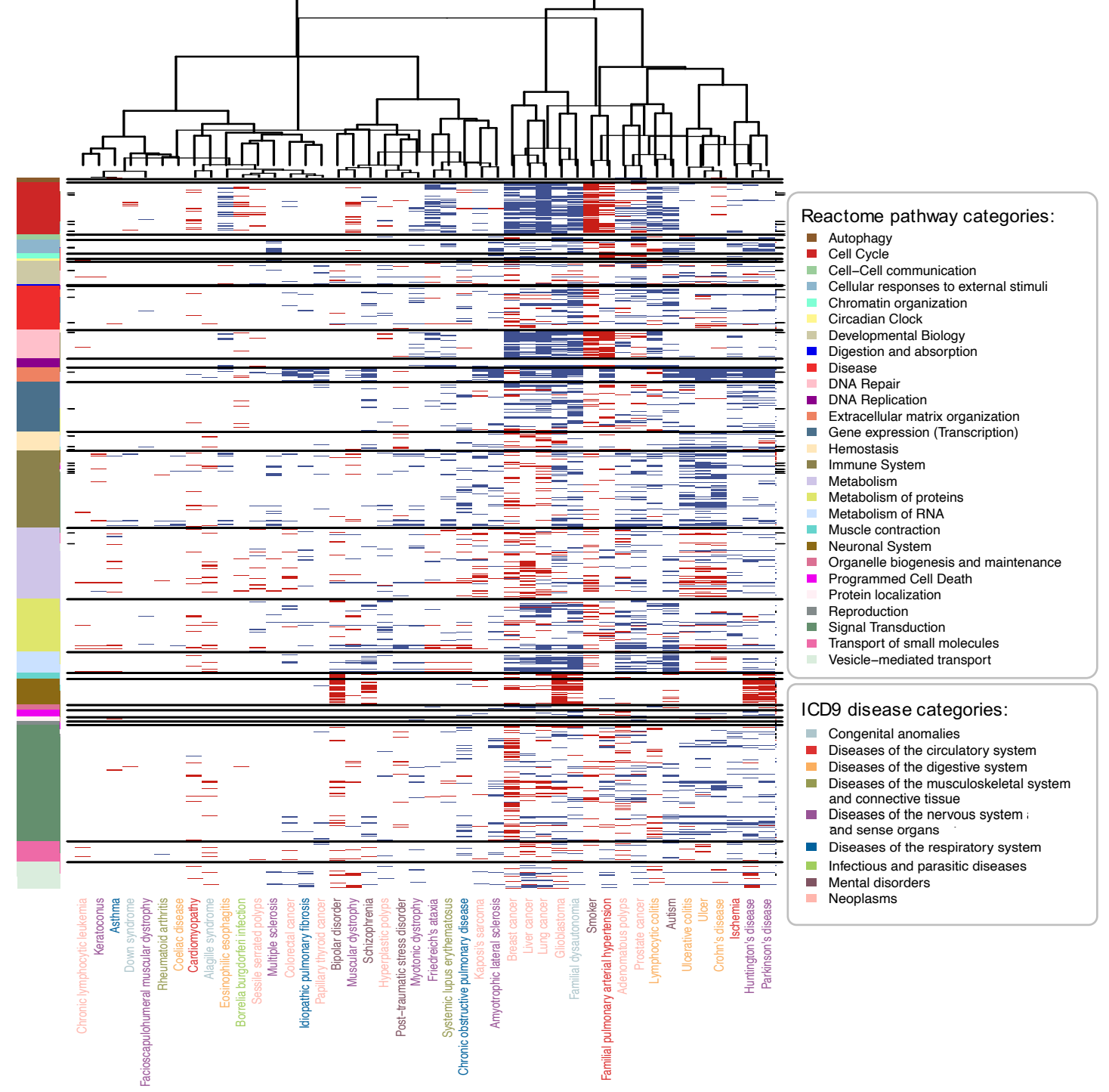

83 Fig. 1. Reactome pathways significantly dysregulated in human diseases grouped by pathway

category. For each disease, Reactome pathways significantly up- and down-regulated were identified using the GSEA method (FDR $<=0.05$ ). Ward2 algorithm was applied to cluster diseases based on the Euclidean distance of their binarized Normalized Effect Size (1s, and -1s for up- and down-regulated pathways). The heatmap shows the dysregulated Reactome pathways (rows) in the diseases (columns), where up- and downregulated pathways are blue and red colored respectively. Reactome pathways are sorted and grouped by pathway categories (separated by black horizontal lines). Diseases are colored by ICD9 disease category.

90 Diseases with 0 dysregulated pathways are not shown.

92 When considering the pathway enrichment (Fig. 1), two main clusters are defined, one containing less enriched pathways than the other. Most ICD9 disease categories have diseases distributed across the

94 branches, pointing to the involvement of some specific shared biological processes in their pathology.

95 Among others, neuronal system- and extracellular matrix (ECM) organization-related pathways are 
medRxiv preprint doi: https://doi.org/10.1101/2021.07.22.21260979; this version posted July 24, 2021. The copyright holder for this preprint (which was not certified by peer review) is the author/funder, who has granted medRxiv a license to display the preprint in perpetuity.

All rights reserved. No reuse allowed without permission.

96

97

98

99

100

101

102

103

104

105

106

107

108

109

110

111

112

\section{Disease similarity network}

114 Next, we built a disease similarity network (DSN) connecting diseases based on the Spearman's 115

116

117

118

119

over and underexpressed respectively in mental and nervous system disorders (bipolar disorder, schizophrenia, Huntington's disease (HD), Parkinson's disease and autism), as previously described in the literature ${ }^{16,17}$. On the other hand, most neoplasms present the expected overexpression of pathways related to the cell cycle, DNA repair, DNA replication, ECM, as well as a decreased hemostasis. Nonetheless, alterations related to developmental biology, immune system and signal transduction are generally observed in specific cancer types ${ }^{18-20}$.

Moreover, diseases of the digestive system like the Inflammatory Bowel Diseases (IBD): Crohn's disease and ulcerative colitis, as well as the closely related lymphocytic colitis and ulcer, present an overexpression of a broad set of immune system-related pathways (Supplementary Fig. 2a). Specifically, ulcerative colitis, Crohn's disease and ulcer form a distinct cluster and share pathways mainly related to cell-cell communication, hemostasis, metabolism, and signal transduction (Supplementary Fig 2b-d and 3a). A common overexpression of multiple specific pathways regarding the ECM organization is observed in IBD (Supplementary Fig. 4c), recently described not only as a consequence of the in situ inflammation but also as an active mediator of $\mathrm{it}^{21}$. Among other pathways, ECM processes are also observed and described to be altered in diseases for which IBD is a risk factor, such as ulcer and colorectal cancer ${ }^{22,23}$. correlation $(\mathrm{FDR}<=0.05)$ of the genes in the union of their sDEGs (see Methods, Fig. 2). The network is composed of one single connected component $-63.37 \%$ positive interactions, $36.63 \%$ negative interactions - with a mean degree of 29.24 (Supplementary Table 2, Supplementary Fig. 5, see Methods). Nodes' degree positively correlates with the number of sDEGs and the sample size of the diseases (Supplementary Fig. 6). 

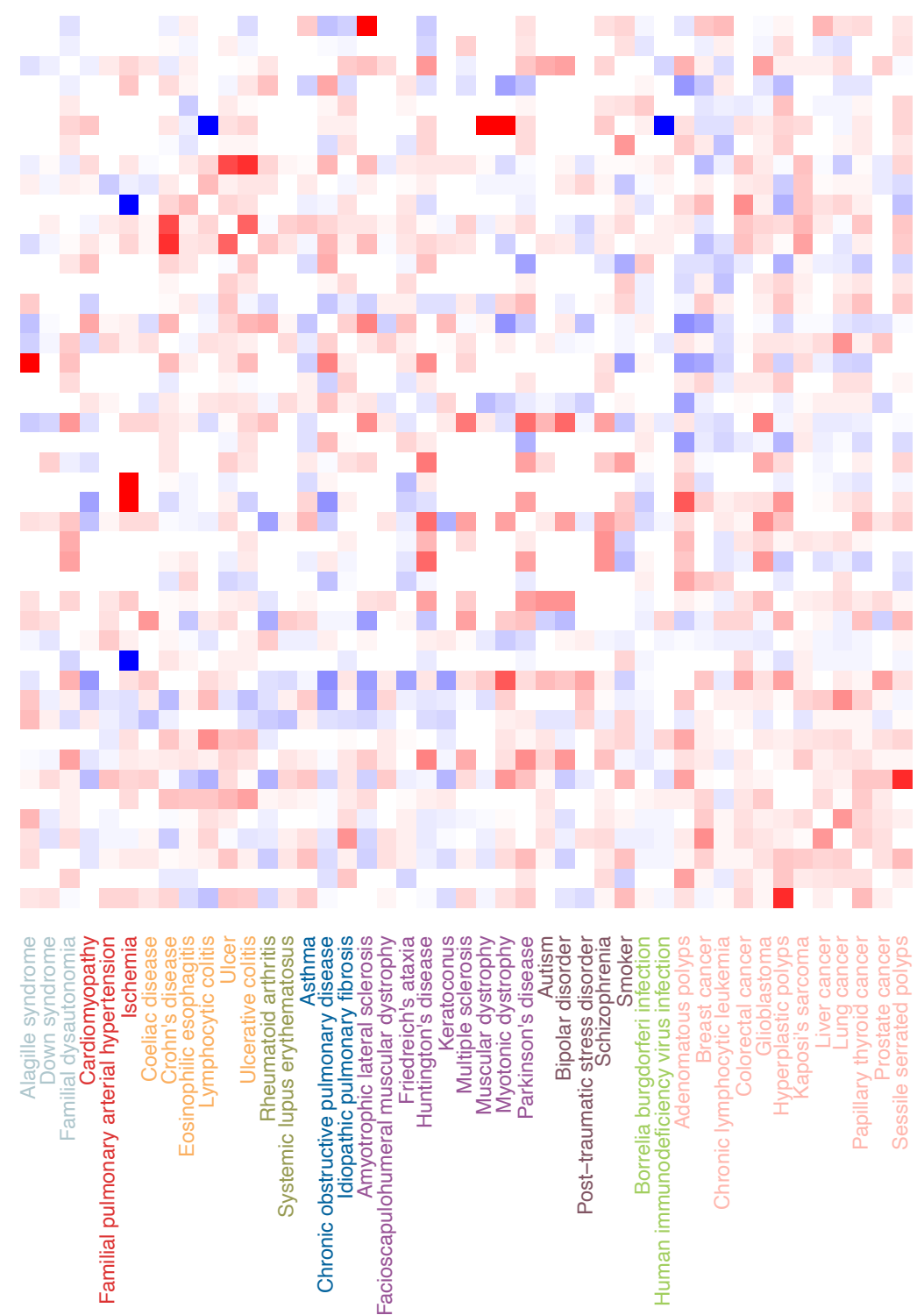

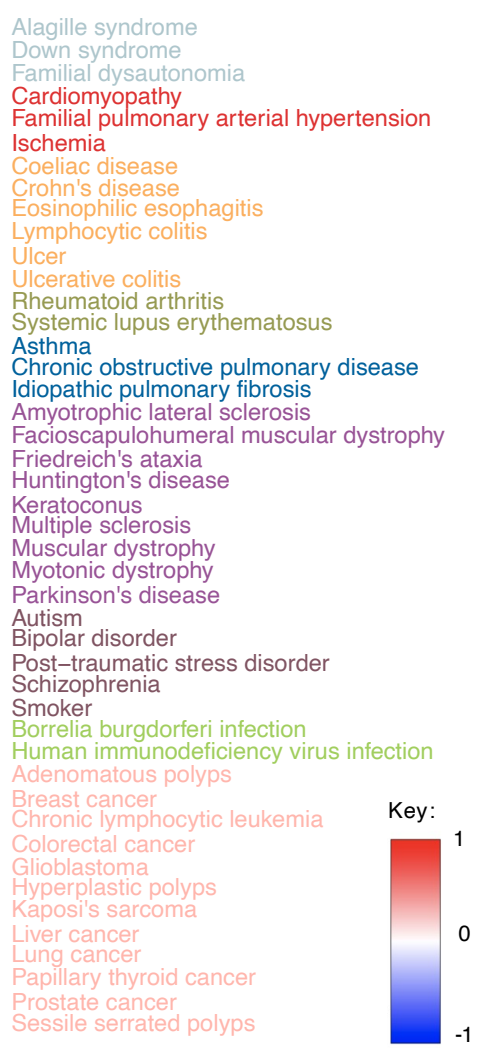

ICD9 disease categories:

- Congenital anomalies

- Diseases of the circulatory system

Diseases of the digestive system

- Diseases of the musculoskeletal system and connective tissue

Diseases of the nervous system and sense organs

- Diseases of the respiratory system

- Infectious and parasitic diseases

- Mental disorders

- Neoplasms

Fig. 2. Heatmap representation of the disease similarity network. Pairwise disease correlations were computed based on the Spearman's correlation of the union of the sDEGs of each pair of diseases. A diseasedisease network was built, containing the significantly positive and negative correlations ( $\mathrm{FDR}<=0.05$ ), where the edge weights in the network correspond to the Spearman's correlations (see Methods). The heatmap shows the network's positive and negative disease interactions, in red and blue respectively. White represents pairs of diseases that are not connected. Diseases are colored by ICD9 disease category.

The DSN captured the previously described IBD comorbidities, as well as intra-disease category comorbidities regarding neoplasms (e.g. lung and liver cancer). We also observed a positive correlation between the differential expression profiles of Kaposi's sarcoma (KS) and human immunodeficiency virus' infection (HIV), being this neoplasm one of the most common malignancies in HIV patients as a 
medRxiv preprint doi: https://doi.org/10.1101/2021.07.22.21260979; this version posted July 24,2021 . The copyright holder for this preprint (which was not certified by peer review) is the author/funder, who has granted medRxiv a license to display the preprint in perpetuity.

All rights reserved. No reuse allowed without permission.

132

133

134

135

136

137

138

139

140

141

142

143

144

145

146

147

148

149

150

151

152

153

154

155

156

157

158

159

160

161

consequence of their immune deficiency ${ }^{24}$. We also captured a previously described less frequent link between KS and other diseases characterized by an immune system imbalance, such as IBD $^{25,26}$.

Many positive interactions entailing diseases of the nervous system and mental disorders are observed, mainly due to shared neurological dysfunction, ECM dysregulation and, in some cases, immune system involvement (Fig. 1 and 2). Among others, schizophrenia was found to be connected to bipolar disorder, autism, and Parkinson's and Huntington's diseases, which are known to be comorbid.

Additionally, we observed some negative correlations between the expression profiles of specific central nervous system disorders (CNSd) and cancers ${ }^{27}$. For instance, HD presents negative correlations with liver, lung, and breast cancer and chronic lymphocytic leukemia, which are known to co-occur less than the expected by chance ${ }^{28}$. When comparing the altered pathways for both diseases, we find opposite molecular tendencies in multiple of their key pathogenic processes. On one hand, cancer is characterized by an overexpression of cell cycle and gene transcription processes, whereas HD shows increased cell death, apoptosis, mitochondrial dysfunction and a negative regulation of gene expression (Supplementary Fig. 3c-d, 2c and 4a). Kinesins-related pathways, involved in cell division and intracellular transport, are overexpressed in cancer as previously described ${ }^{29}$, and underexpressed in $\mathrm{HD}$, where its impairment is also characterized ${ }^{30}$ (Supplementary Fig. 2c). Additionally, immune abnormalities have been extensively described as central to HD and cancerous processes ${ }^{31,32}$. For instance, we observed an increased interleukin production and signaling regarding Th1-type immune response (e.g. IL-12) in HD, as well as an activation of the complement cascade, being both processes underexpressed in cancer and previously linked to carcinogenesis ${ }^{31-33}$ (Supplementary Figure 2a).

Subsequently, we evaluated to what extent the DSN is able to capture medically known comorbidities by computing its overlap with the epidemiological network from Hidalgo et $a l^{2}$. We observed that the DSN significantly overlaps $46.2 \%$ of the interactions in Hidalgo et al. ${ }^{2}$ over the common set of diseases $(p$-value $=0.0018)($ Supplementary Table 3 , see Methods). We also showed that the overlap of the negative interactions was not significant $(\mathrm{p}$-value $=0.867)$. The DSN precision and recall varies depending on the disease category pair (Fig. 3a). For instance, diseases of the digestive system present the highest precision $(66.4 \%)$ with a mean recall of $53.6 \%$. Interactions entailing congenital anomalies are also captured at a high level. On the contrary, highly heterogeneous diseases (e.g. mental disorders) tend to present lower recall values and neoplasms, which often share the dysregulation of multiple pathways without being comorbid, exhibit the lowest precision. 


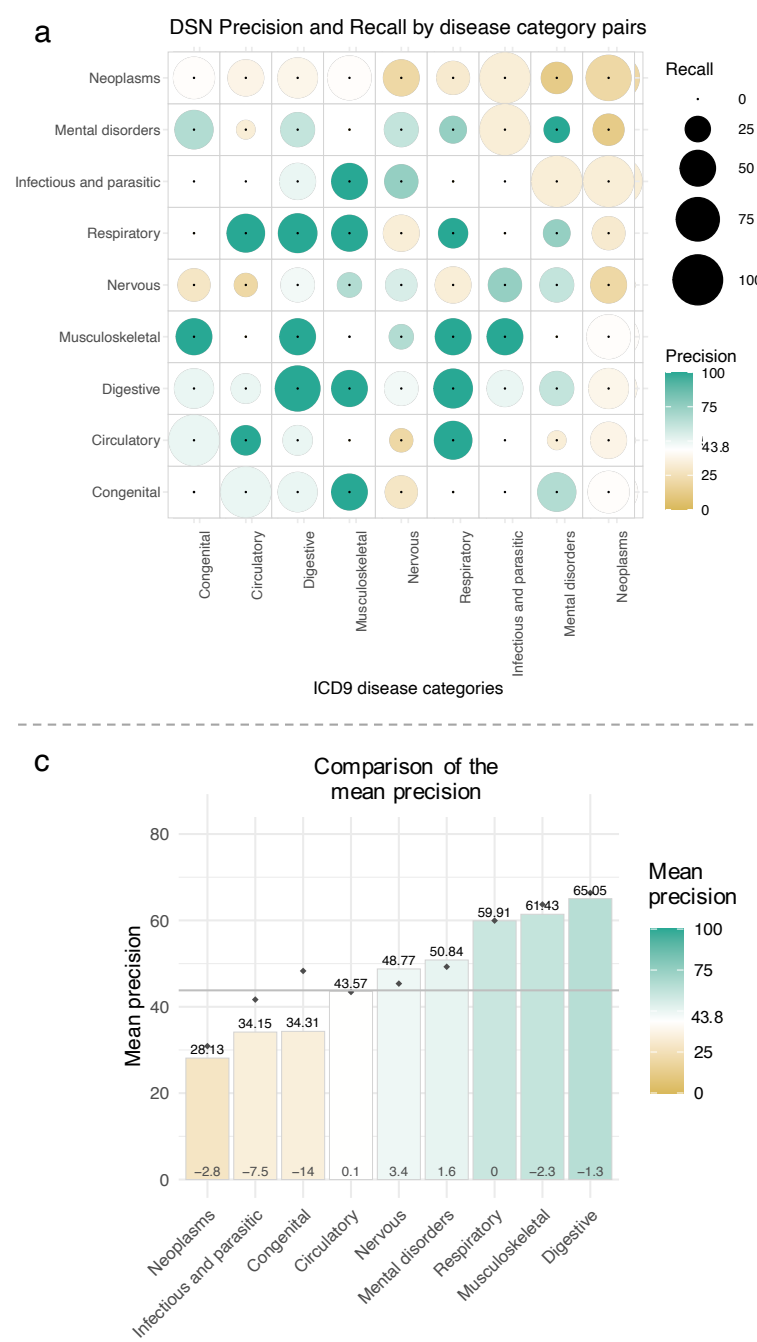

ICD9 disease categories

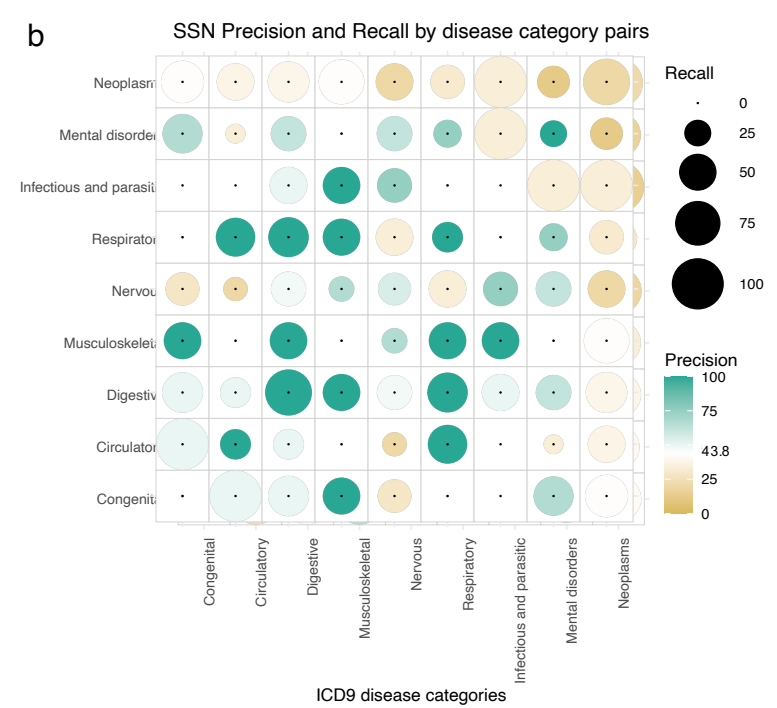

d

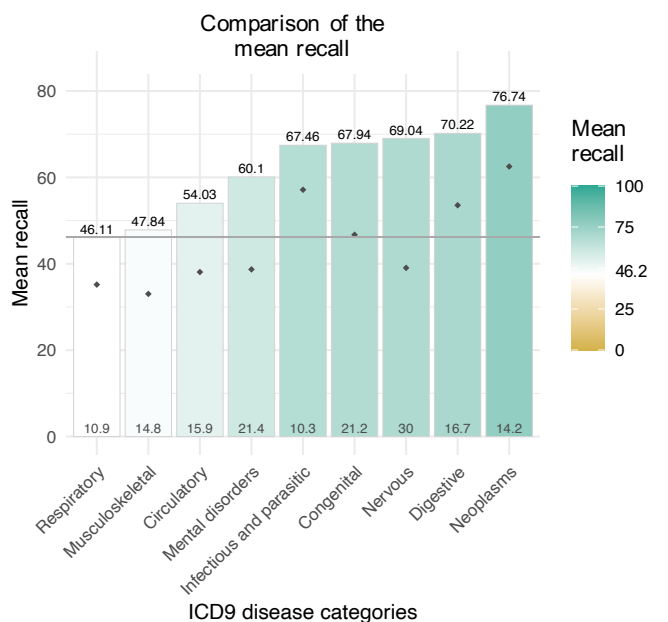

Figure 3. Precision and recall of the DSN and SSN by disease category. Precision and recall of the (a) Disease Similarity Network (DSN) and the (b) Stratified Similarity Network (SSN) by disease category pairs. The precision is the percentage of interactions in the molecular networks present in the epidemiological network from Hidalgo et $a .^{2}$ and the recall is the percentage of epidemiological interactions captured by the molecular networks. For the SSN, interactions between meta-patients and diseases were considered (See Methods). Each point in the symmetric matrix corresponds to the subnetwork that results from selecting the interactions between diseases of the indicated disease categories. The area of the circles represents the recall and the color corresponds to the precision. Green and yellow colors indicate higher and lower precisions than the one of the DSN (43.8\%), represented in white. Disease pairs without epidemiological interactions present a single black point. (c) Mean precision of each disease category in the SSN. Disease categories are sorted by their mean precision. Green and yellow colors indicate higher and lower precisions than the one of the DSN, represented in the horizontal grey line. (d) Mean recall of each disease category in the SSN. Disease categories are sorted by their mean recall. Green and yellow colors indicate higher and lower recalls than the one of the DSN (46.2\%), represented in the horizontal grey line. Black points indicate the mean (c) 
precision or (d) recall of the disease categories in the DSN. The differences between the values at the metapatient and disease level are plotted in the base of the bars.

179

Moreover, we studied the topological properties of the molecular and epidemiological networks 181 (Supplementary Table 4). Both networks contain one single connected component that comprises all 182 the diseases, being the DSN denser. The DSN composed only of positive interactions and the epidemiological network present a transitivity around 0.5 , meaning that nodes connected to a third one have 0.5 probability of being connected. Also, both networks present a mean distance below 2 , indicating a high connectivity. Then, we compared the topological properties of the positive ICD9based DSN subnetwork and the epidemiological network, considering only the common set of diseases (Supplementary Table 5). We observe that both subnetworks have a very similar global topology. For instance, they present a significantly equal mean degree and a very similar density and mean distance (slightly higher for the epidemiology) around 0.42 and 1.6 respectively. Although both networks have a similar mean transitivity above 0.5 , it is significantly higher for the epidemiological network, possibly due to other forces such as common risk factors or lifestyle. The networks also present a significantly equal mean closeness, betweenness and degeneracy. Finally, we computed the assortativity of the networks labeling the nodes with their ICD9 disease category. We found that both networks present an assortativity around 0 ; the epidemiological network is slightly depleted in within-category disease links whereas the DSN seems to present a minimal enrichment in those.

Next, we compared our overlap with the ones derived from published disease-disease networks based on other molecular data (see Methods). Both the microbiome ${ }^{10}$ and the miRNA ${ }^{9}$ networks yielded nonsignificant overlaps with the epidemiological network over their respective common diseases and over the common diseases also present in the DSN. The network derived from protein-protein interaction (PPI) networks presented significant yet small overlaps with the epidemiology $(8.71 \%$ for the entire network and $18.52 \%$ over the diseases in the DSN), and the one generated by Sánchez-Valle et al. ${ }^{8}$ using microarrays presents a significant overlap of $16 \%$ with the epidemiological network from Hidalgo et $a .^{2}$ (Fig. 4a and Supplementary Table 6).

204 Finally, we compared the networks that present a significant overlap with the epidemiology (PPI and microarray-based networks) with the DSN (Supplementary Table 7-8). The network derived from PPIs and the DSN share 19 ICD9 codes and only 6 out of the 20 interactions present for these diseases in the

207 former are found in the later (there is no significant overlap between them) (Supplementary Table 7-8). Between these common diseases, 29 epidemiologically known comorbidities are connected only in the 
DSN whereas 10 are unique to the other network (Fig. 4b). The entire DSN provides information for 22 new ICD9 codes and uniquely captures 149 disease links described in the epidemiology.

211 On the other hand, the microarrays' network contains 92 ICD9 codes, where 27 of them are analyzed 212 in the DSN. We computed the overlap of both networks over the common set of ICD9 codes, yielding 213 a significant overlap (p-value $=0.027$ ) of $47.02 \%$ of the microarrays' network. Specifically, positive 214 interactions have a significant overlap $(p$-value $=0.002)$ of $62.22 \%$ whereas the overlap of the negative 215 interactions is not significant $(\mathrm{p}$-value $=0.624)($ Supplementary Table 8$)$. Among these common 216 diseases, the DSN yielded 42 new positive interactions that are described in the epidemiological 217 network by Hidalgo et al. ${ }^{2}$ (e.g. Crohn's disease and ulcerative colitis) (Fig. 4c). Additionally, the DSN 218 provides information for 14 new ICD9 codes and captures 141 new interactions that match known 219 comorbidities.

a

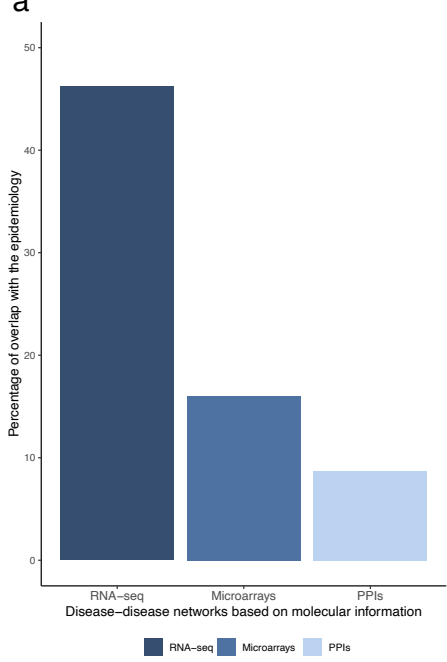

b

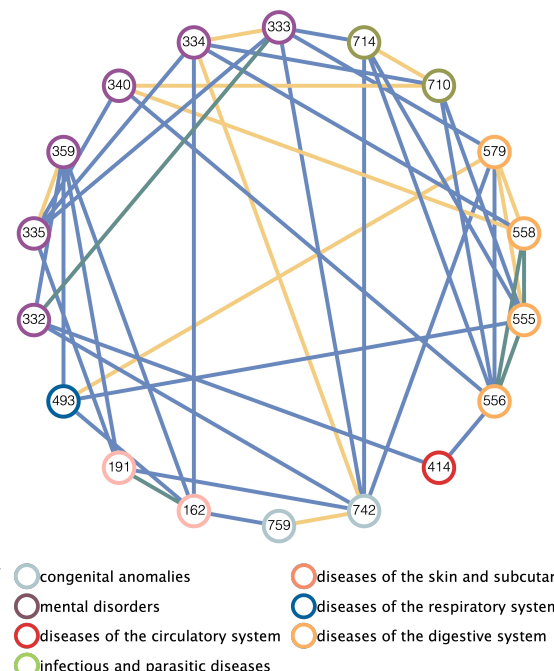

C

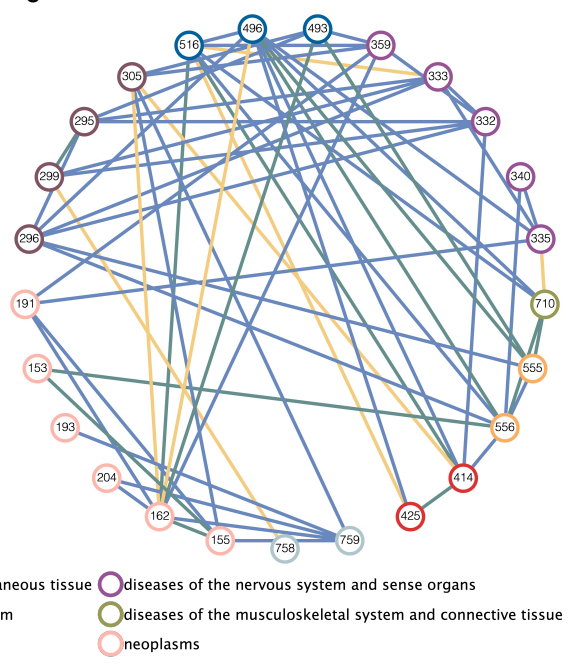

Figure 4. Comparison of the epidemiological interactions described in the Disease Similarity Network interactions in the epidemiological networks, significantly captured by molecular networks. It shows the overlap of our DSN based on RNA-seq and the networks based on microarrays and protein-protein interactions (PPI) (See Methods).

(b) Network visualization of the positive disease-disease interactions described in the epidemiological network by Hidalgo et al. ${ }^{2}$ that captured only by the DSN (blue), only by the network based on PPIs ${ }^{6}$ (yellow) or by both of them (green). It shows the interactions over the common set of ICD9 codes in both molecular networks. Diseases are colored by their ICD9 code category. 
by Hidalgo et $a .^{2}$ that captured only by the DSN (blue), only by the DMSN based on microarrays ${ }^{8}$ (yellow) or by both of them (green).

233

\section{Molecular mechanisms behind comorbidities}

235 Once confirmed that the DSN captures a significant percentage of comorbidities, we inspected which 236 are the molecular mechanisms underlying its epidemiological (EIs) and non-epidemiological 237 interactions (NEIs), i.e. interactions for which it was not possible to find a correspondence with the 238 current medical data. We observe that the Reactome pathway categories behind most interactions tend to display a wider range of dysregulation than those affected only in some interactions; i.e. they share

240 a higher number of altered pathways (Fig. 5). Impressively, 95.2\% of the EI in the DSN share at least 241 one -and a mean of 21.2- overexpressed immune system pathways, followed by pathways related to the

242 ECM, metabolism of proteins, metabolism and signal transduction, all involved in over $90 \%$ of the interactions $($ means $=10.9,10.1,6.3,16.1)$. The underexpressed pathways involved in most of the EIs

244 are related to the metabolism of proteins, signal transduction, metabolism, and developmental biology

$245 \quad($ means $=4.9,7.2,13.5,2.5)$.

246 To adequately detect which pathways are specific to EI, we also take into account the ratio between the 247 mean number of shared over or underexpressed pathways by Reactome category in EIs versus NEIs

248 (Fig 5, Supplementary Fig. 7). Overexpressed circadian clock pathways explain more EIs than NEIs, 249 being the mean number of shared pathways 1.7 times higher in the former than in the latter. Digestion 250 and absorption and protein localization are 3.8 and 1.6 times more commonly underexpressed in the 251 EIs. 

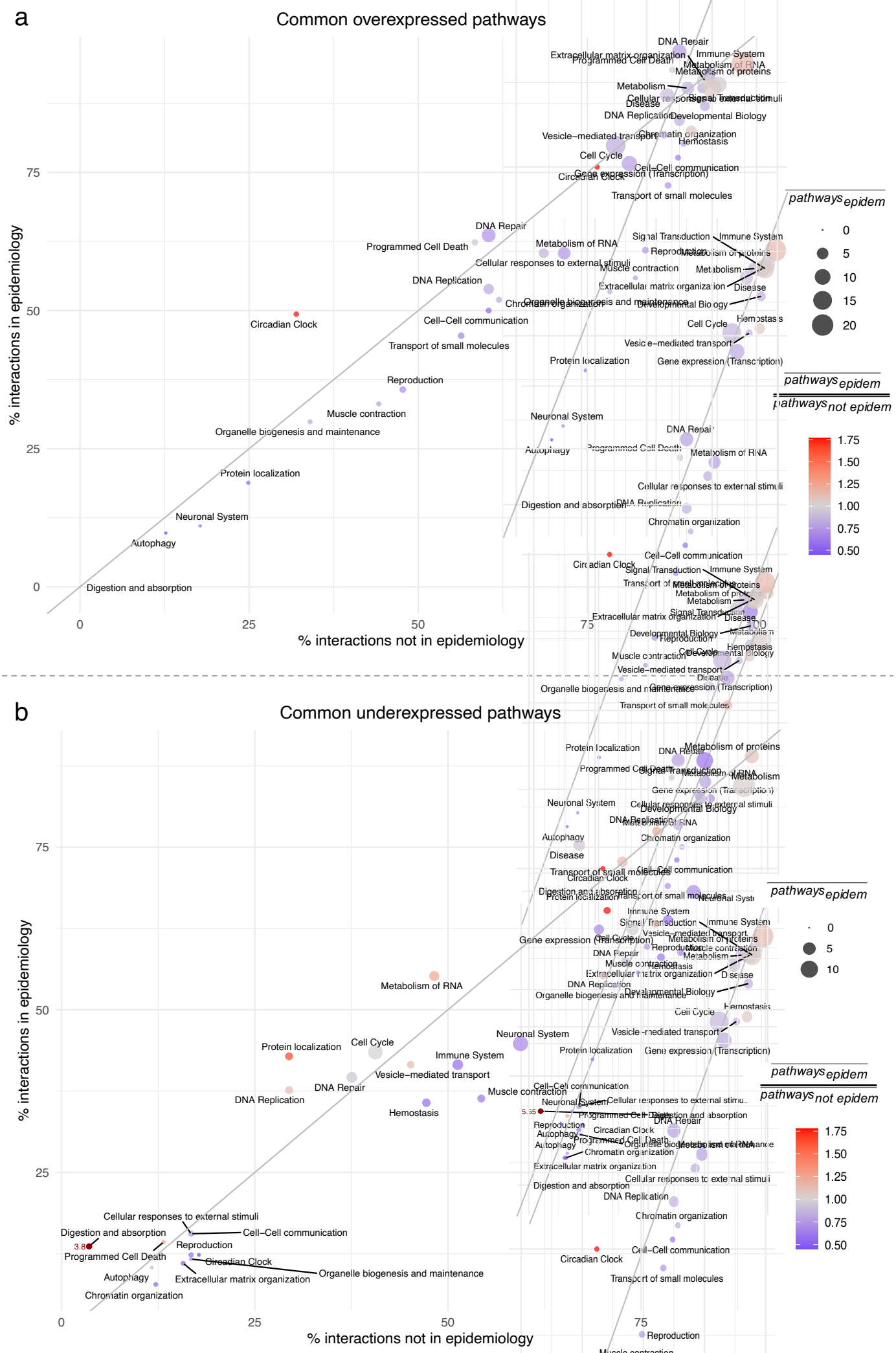

253 Figure 5. Over and underexpressed pathways behind epidemiological and non-epidemiological

254 interactions. Percentage of epidemiological versus non-epidemiological interactions that share (a) 255 overexpressed or (b) underexpressed pathways. Each point represents a Reactome pathway category. The size of the points corresponds to the mean number of shared pathways in the epidemiological interactions. 
The color corresponds to the ratio of the mean number of shared pathways in epidemiological versus nonepidemiological interactions (e.g. red indicates that epidemiological interactions share more altered pathways than non-epidemiological interactions).

260

261 Higher resolution results can be obtained by considering interactions between pairs of disease 262 categories. For instance, the shared overexpression of circadian clock pathways seems to be highly specific of EIs entailing CNSd (Fig. 5 and 6a). Actually, the pivotal and putatively causal role of the circadian system in CNSd and their comorbidities has recently been proposed ${ }^{34-36}$. Although each individual comparison has its particular portrait of the mechanisms underlying disease interactions, some general patterns become apparent. We observe that pathways tend to cluster according to their ability to explain EI versus NEIs. In Fig. 6a we can see a cluster of pathways whose dysregulation is shared by all EIs whereas smaller clusters are mostly present in NEIs. Interestingly, pathway categories involved in more EIs than NEIs also present a higher number of pathways commonly dysregulated in EIs than in NEIs (Fig. 6a, within the upper cluster, redder dots are observed at the left side). In summary, EIs have been found to present more shared altered pathways than NEIs. In fact, if we remove neoplasms, EIs share the alteration of $53.1 \%$ and $56.8 \%$ more over and underexpressed pathways, respectively. Nonetheless, by inspecting the interactions between neoplasms, we can discern between

274 the mechanisms that are potentially responsible for their common cause from the ones that are more 275 likely due to the convergence of some common functions (i.e. overexpression of pathways related to 276 the immune system or cell-cell communication as well as the underexpression of developmental biology 277 or DNA repair processes) (Fig. 6b and Supplementary Fig. 8). Comorbid neoplasms tend to share a higher number of overexpressed pathways related to organelle biogenesis and maintenance, chromatin organization or cell cycle and an underexpression of pathways such as: metabolism, transport of small molecules or the immune system. Interestingly, around $30 \%$ of comorbidities within neoplasms share a highly specific overexpression of protein localization pathways. 
a

Congenital - Nervous

Common overexpressed pathways

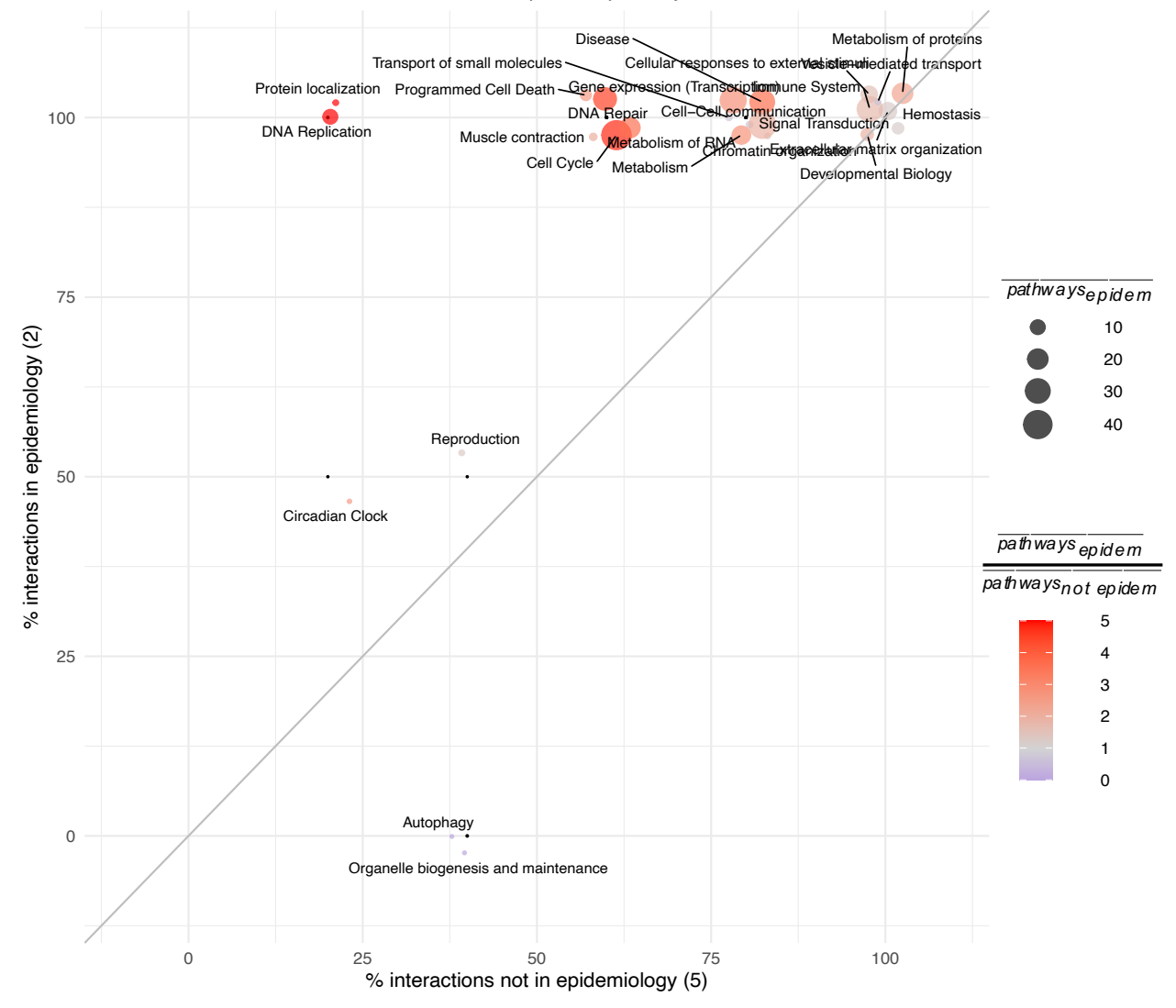

b

Neoplasms - Neoplasms

Common overexpressed pathways

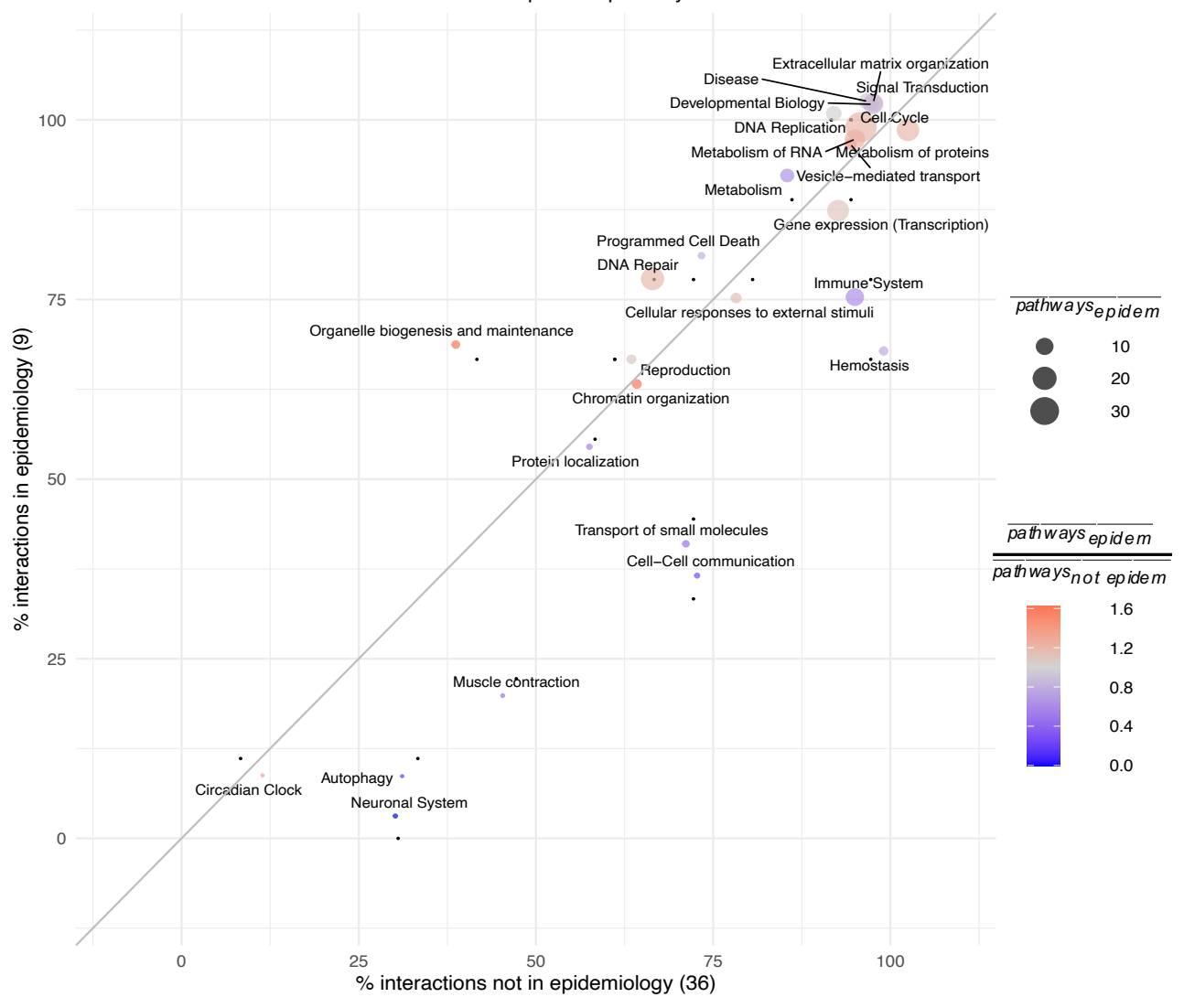


interactions. Percentage of epidemiological (EIs) versus non-epidemiological interactions (NEIs) that share overexpressed pathways. Each point represents a Reactome pathway category. The size of the points corresponds to the mean number of shared overexpressed pathways in the EIs. The color corresponds to the ratio of the mean number of shared pathways in EIs versus NEIs (e.g. red indicates that epidemiological interactions share more pathways than non-epidemiological interactions). The number of EIs and NEIs is indicated between parentheses in the $\mathrm{y}$ and $\mathrm{x}$ axis labels, respectively.

(a) Interactions between congenital anomalies and diseases of the nervous system and sense organs. (b) Interactions within neoplasms.

\section{Defining disease meta-patients}

293 It has been shown that patients suffering from a given disease often present different risks of developing specific secondary conditions ${ }^{4}$. We hypothesize that such differential risks might be driven by the existence of disease subtypes. To evaluate it, we studied disease similarities in a stratified manner by applying clustering algorithms to obtain subgroups of cases with a similar expression profile for each disease (see Methods). We consider those subgroups as meta-patients. To evaluate our approach, we selected breast cancer, a disease with known molecular subtypes for which we have two independent studies. The first study contains 20 estrogen positive $(\mathrm{ER}+)$ samples and 18 triple negative $(\mathrm{TN})$ and

300 the second study entails 9 estrogen negative (ER-) samples and 9 ER+. Our two independently obtained 301 clusters (using $\mathrm{PAM}^{37}$ and Ward2 ${ }^{38}$ clustering algorithms separately) yielded similar results, being PAM the most accurate when grouping cases according to their defined molecular subtypes (Supplementary

303 Fig. 9 and Supplementary Table 9). Breast cancer patient's clustering and pairwise similarity shows 304 that PAM clustering classifies most of the cases correctly into estrogen negative (ER-), triple negative 305 (TN) and estrogen positive (ER+) (Fig. 7a), even grouping cases with shared molecular subtypes that belong to two independent studies. 

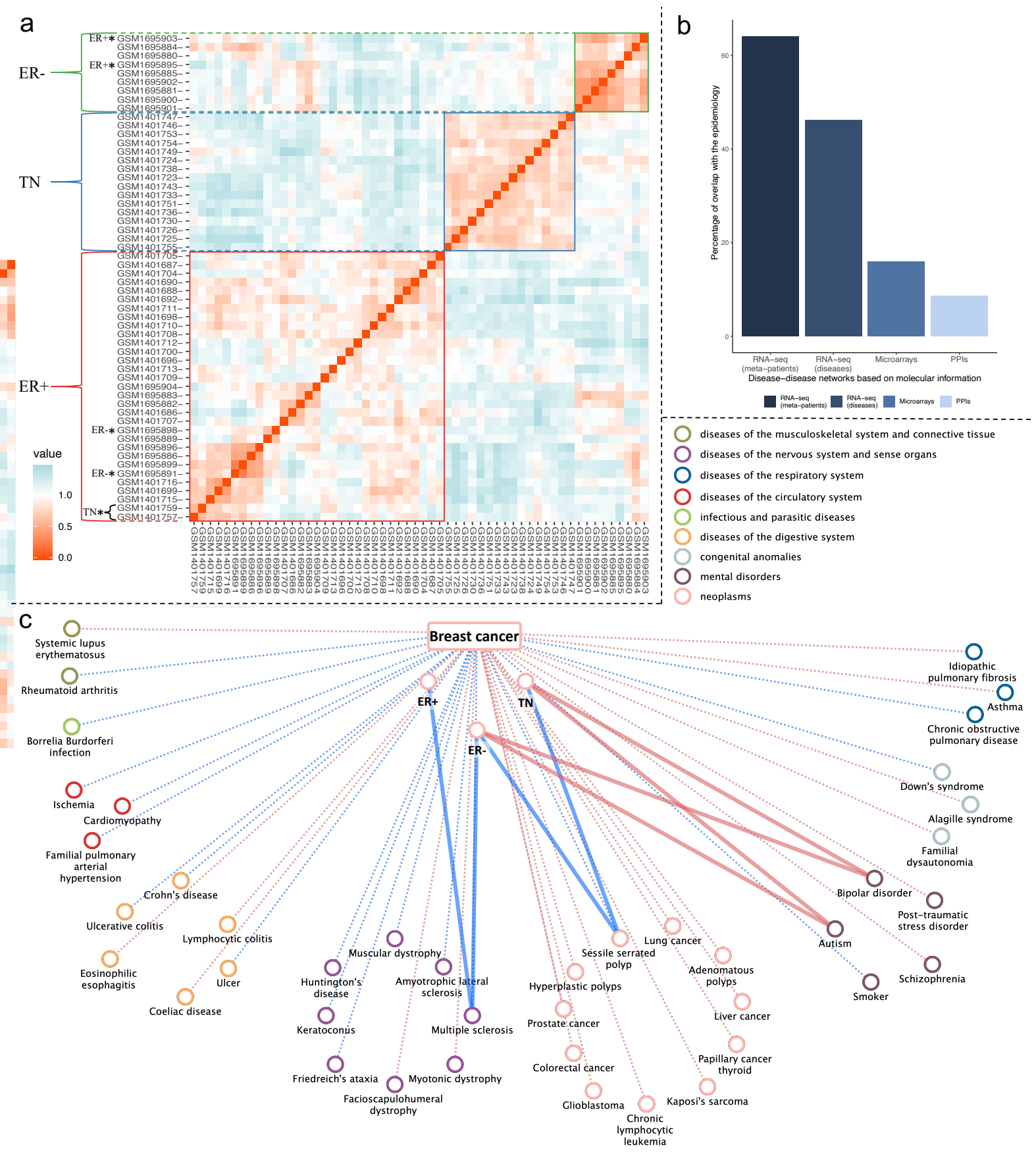

Fig. 7. Breast cancer meta-patients and breast cancer molecular similarity interactions at the disease and meta-patient level. (a) Heatmap showing breast cancer patients' similarity and classification through PAM algorithm. The similarity has been defined as $1-$ Spearman's correlation of the gene expression values.

311 Orange represents a positive correlation between the expression profile of the patients whereas blue represents a negative correlation between them. Patients are colored based on their molecular subtype.

313 Patients are divided in 3 subtypes: ER- (Estrogen negative) in green, TNEG (Triple negative patients) in

314 blue and ER+ (Estrogen positive) in red. Patient's clustering is marked on the left side of the figure. (b)

315 Percentages of interactions in the epidemiological networks, captured by molecular networks in a significant manner. It shows the overlap of the interactions at the meta-patient and disease level based on RNA-seq and 
the networks based on microarrays and protein-protein interactions (PPI) (See Methods). (c) Interactions between breast cancer disease and its meta-patients with human diseases. Positive interactions are represented in red and negative interactions in blue. Dashed-faded lines represent interactions at the disease level and shared by all the meta-patients whereas solid lines represent meta-patient specific interactions.

321

\section{Stratified Similarity Network}

323 Since meta-patients are groups of patients from a given disease, they can be treated as any phenotype 324 to which we can apply gene expression analysis methods that assign a significance to their conclusions.

325 Hence, and first of all, we characterized the obtained meta-patients in terms of their differential 326 expression profiles (see Methods). Then, we built a network based on the gene expression similarity between all the meta-patients and analyzed diseases following the methodology described for the generation of the DSN (see Methods). The resulting Stratified Similarity Network (SSN) contains three types of interactions: (1) the previously described disease-disease interactions, (2) interactions connecting different meta-patients and (3) interactions connecting meta-patients to diseases. The SSN can be fully explored in the web application (see Methods) and its topological properties can be found in the Supplementary Table 4. Since the SSN has a considerably higher number of nodes, we observe a large increase in mean degree with respect to the DSN. As captured by the moderate increase in density and mean transitivity, the meta-patients have added some new interactions that were missed by the DSN, being able to uncover new transitive relationships. These properties, along with a concordant decrease in diameter and mean distance, occur across the entire network and subnetworks entailing positive or negative interactions. In fact, by defining meta-patients we significantly increase the number of diseases linked to each disease by 7.64 diseases $(p$-value $=1.596 \mathrm{e}-10)$ and $7.29(\mathrm{p}$-value $=6.159 \mathrm{e}-15)$ for the positive and negative interactions respectively (Supplementary Fig. 10a). We confirmed that this increase in detection power is significant compared to randomly generated meta-patients ( $p$-value $=0$ for positive and negative interactions) (see Methods) (Supplementary Fig. 11).

342 We inspected the links between breast cancer disease and its meta-patients with the rest of diseases (Fig.

$3437 \mathrm{c}$ ). We observe that while most of the interactions are shared between breast meta-patients and the 344 disease, several interactions are specific to some of them. For instance, a negative interaction with 345 multiple sclerosis is only found in ER+ and ER- meta-patients while a positive interaction with autism 346 and bipolar disorder is specific to TN and ER- meta-patients.

347 While breast cancer meta-patients share most of their connections, this is not the case for all the diseases.

348 Actually, the percentage of positive and negative links shared by all the meta-patients from a given 
349 disease varies greatly (Supplementary Fig. 10b). For example, CNSd (e.g. schizophrenia, bipolar

350 disorder, MS or autism), known to be highly heterogeneous, show little consistency in their meta-

351 patients' interactions. On the other hand, neoplasms - that tend to present a consistent and high alteration

352 of multiple biological processes - seem to present a higher correspondence in their meta-patients' 353 connections.

354 To evaluate the ability of meta-patients to uncover new comorbidities, we computed the overlap of the

355

356

357

358

359

360

361

362

363

364

365

366

367

368

369

370

371 So far, many molecular representations of disease interactions have failed to explain a noteworthy 372 interactions between meta-patients and diseases with the epidemiological data (see Methods). Remarkably, meta-patients significantly captured $64.1 \%(p$-value $=0.0187)$ of the interactions in the epidemiological network from Hidalgo et al. $^{2}$, which corresponds to an increase in recall of $17.9 \%$ with respect to the DSN with a very small decrease in precision of $0.7 \%$. On the contrary, negative interactions do not show a significant overlap $(\mathrm{p}$-value $=0.8035)($ Supplementary Table 10). As Fig. 3 shows, most disease categories benefit from the stratification of diseases, since they tend to present a considerable increase of the recall which is usually accompanied by a slight decrease in precision. Aligned with previous results, highly heterogeneous diseases (nervous system and mental disorders) present some of the highest increases in recall (up to 30\%); strikingly, also gaining precision. More moderately, this tendency also occurs for circulatory diseases. Respiratory diseases, which often display a wide range of immune system responses, present $10.9 \%$ more recall with the same precision than in the DSN.

Finally, we developed a web application (http://disease-perception.bsc.es/rgenexcom/) in which the networks and their underlying molecular mechanisms can be easily inspected (see Methods).

\section{Discussion} number of the medically known comorbidities, being unable to answer the long-standing question of the molecular origin of comorbidities. The generated networks based on RNA-seq profiles provide a convincing and comprehensive answer to this matter, being able to significantly capture and meaningfully explain $64 \%$ of the known comorbidities. Hence, they render a qualitative difference over previous studies, providing a solid support to the key role of molecular mechanisms behind comorbidities in a generalized manner.

Actually, the DSN and the epidemiological network are very similar from a topological perspective. They present significantly equal mean degree, closeness, betweenness and degeneracy, as well as very 
380

381

382

383

384

385

386

387

388

389

390

391

392

393

394

395

396

397

398

399

400

401

402

403

404

405

406

407

408

409

410

411

similar density and mean distance; indicating a general resemblance in their overall structure and information flow. They also show close to zero assortativities with respect to disease categories (minimally negative in the epidemiology and positive in the DSN), which implies a slight tendency of diseases from the same category to be linked more than expected in epidemiology. For instance, while the observed molecular similarities connect some neoplasms that are indeed comorbid (e.g. liver cancer with glioblastoma, lung, or colorectal cancer), they also link specific cancers that are not epidemiologically linked. This evidence shows that the presence of shared molecular mechanisms does not always translate into an increased relative risk that is observed in the currently limited medical data. However, we can discern between the mechanisms behind well-established comorbidities from the ones that may be a consequence of an overall molecular similarity, which is especially relevant for neoplasms that share similar dysregulated pathways. Indeed, without considering neoplasms, EIs tend to share over $50 \%$ more altered pathways than pairs of diseases without clear evidence of a medical relation (NEIs).

Several ways in which shared molecular mechanisms can underlie direct comorbidities have been proposed. Essentially, molecular mechanisms can be causally or consequentially altered in a given disease; which, in turn, can contribute to the development of a secondary condition. Thus, molecularly based comorbidities can be explained by the following scenarios: (1) both diseases share the same or correlated causal alterations, (2) the molecular mechanisms altered as a consequence of one disease are associated to the second condition or (3) there is a third condition that increases the risk of developing both of them ${ }^{1}$. These schemes are not mutually exclusive and can be combined in complex manners. In fact, the study of direct comorbidities in longitudinal studies has shown disease trajectories that can be explained by an underlying aggravation and accumulation of specific molecular processes, especially in a chronic manner ${ }^{39}$. This is the case for the discussed progression of IBD into colorectal cancer ${ }^{40}$ and for the highly prevalent disease trajectory that has recently been called metabolic syndrome, including obesity, insulin resistance, diabetes, cardiovascular disease or even cancer ${ }^{41}$. These observations supported the central role of the underlying molecular mechanisms in the study of individual diseases and disease comorbidities, to the point where efforts have already been destined to redefining diseases by incorporating both their clinical features and molecular profiles ${ }^{42}$.

Comorbidity relationships can be better understood if disease subtypes and patient-specific patterns are taken into account ${ }^{4}$. Indeed, previous epidemiological studies have identified comorbidities that depend on the disease subtype ${ }^{43-45}$. In line with this, we introduced the concept of meta-patients and the stratified exploration of their molecular similarities with diseases. The definition of meta-patients unraveled a significant mean of around 14 new subgroup-specific disease connections per disease, 
412 increasing the detection power of disease similarities (Supplementary Fig. 10a). This subclassification

413 of diseases based on similarities of the patients' gene expression profiles can be related to

414 epidemiological observations of comorbidities that depend on patients' characteristics. In the case of

415 breast cancer, we observed that although the three meta-patients share most of the disease interactions,

416 some specific and interesting ones emerge. For instance, TN and ER- meta-patients are the only ones

417 presenting a positive interaction with autism and bipolar disorder (Fig. 7c). While several studies ${ }^{46,47}$

418 have found no significant correlations, recent molecular and epidemiological evidence suggests cancer

419 as a comorbidity of autism ${ }^{48,49}$. Besides, an enhanced cancer risk has been described for bipolar disorder

420 patients in both genders, being the risk for breast cancer higher but non-significant ${ }^{50}$. Additionally, we

421 observed a negative interaction between breast cancer, ER+ and ER- meta-patients with multiple

422 sclerosis (Fig. 7c). Again, opposite tendencies are described in the literature for this connection, where

423 the order of appearance of the disease seems to drive the comorbidity pattern. It has been shown that

424 breast cancer patients are $45 \%$ less likely to develop multiple sclerosis ${ }^{51}$. On the other hand, multiple

425 sclerosis patients have been shown to have a significantly increased risk of breast cancer, presumably

426 driven by immunosuppression derived from the associated treatment ${ }^{52}$. Therefore, our analysis provides

427 new evidence on subgroup-specific comorbidities with a potential molecular explanation. Moreover,

428 we showed that the percentage of recapitulated epidemiological interactions increased from almost half

429 to $64.1 \%$ when considering the interactions between diseases and meta-patients, with a slight decrease

430 in precision of $0.7 \%$.

431 As shown, the generated networks provide the first systematic translation of disease comorbidities into

432 molecular patterns. Previous efforts based on disease-associated genes in a PPI network were limited

433 by the incompleteness of the interactome and the biased knowledge of disease-associated genes for

434 highly studied diseases ${ }^{6}$. Furthermore, networks based on other molecular sources such as the

435 microbiome $^{10}$ and miRNAs ${ }^{9}$ do not overlap epidemiological interactions significantly.

436 There are at least three factors that allowed us to significantly improve the explainability of known

437 comorbidities captured with gene expression information. First, the better quality and coverage of RNA-

438 seq data, that unbiasedly increases the number and quality of features whose similarity can be compared

439 between diseases. Secondly, an improved methodology based on the existence of a significant

440 correlation between the differential gene expression profiles of each disease pair. As a result, our disease

441 links are robust, stable and independent of the rest of diseases, even if the disease universe changed;

442 contrary to previous attempts based on relative molecular similarities, where disease links depended on

443 the rest of the network ${ }^{8}$. Finally, the stratification of diseases into subgroups of cases named meta- 
444 patients. Opposite to patient-centric approaches, meta-patients can be methodologically treated as 445 phenotypes are handled in gene expression studies; thus, a significance can be associated to their altered 446 genes, pathways and, importantly, disease interactions in the SSN.

447 Still, there are a number of issues that, if addressed, could improve the quality of the results and the 448 coverage of the comorbidity space. First, the generated networks contain both positive and negative 449 interactions potentially representing direct and inverse comorbidities. While it is possible to validate

450

451

452

453

454

455

456

457

458

459

460

461

462

463

464

465

466

467

468

469

470

471

472

473

474

475 the positive ones, it is more difficult to validate the less abundant - but equally interesting- negative ones $(36.63 \%)$ since they are not systematically described in large studies and are only sporadically addressed in the literature ${ }^{53}$. Nonetheless, we detected known inverse comorbidities such as: HD and specific cancer types or Parkinson's disease and rheumatoid arthritis ${ }^{54}$. Therefore, a current limitation in this study is the lack of epidemiological networks entailing inverse comorbidity relationships.

Another limitation is the lack of sample information, such as age, sex, or treatments, which may drive transcriptomic differences between patients. Also, our samples belong to published studies focused on a specific disease in a given tissue (e.g. brain, liver or blood). Since we have cases and controls for each study and disease, we were able to correct for the tissue effect when generating sDEGs at the disease and meta-patient level. However, it would be optimal to have comprehensive data sets of diseases from the same tissue or an array of interesting tissues. Moreover, better defined and annotated disease subgroups as well as their differential comorbidities could help us refine the definition of meta-patients and increase their power to capture their epidemiological associations. We observed that patient stratification is specially important for highly heterogeneous diseases. While some diseases (e.g. breast cancer) showed few links specific to some meta-patients, more heterogeneous diseases (e.g. CNSd like schizophrenia or bipolar disorder and immune system disorders like asthma) present a majority of metapatient specific links (Supplementary Fig. 10b).

Future perspectives include increasing sample size, so sex-specific disease similarities can be extracted and compared to their epidemiologically described disease interactions ${ }^{55}$. Furthermore, the molecular coverage of disease comorbidities could be improved by considering other molecular information that may underlie comorbidities within an integrative approach ${ }^{56}$. For this, large disease cohorts comprising different kinds of omics as well as clinical information would be needed. Furthermore, the obtained molecular similarities could be used to guide drug repurposing and development ${ }^{57}$.

In summary, we built disease similarity networks based on transcriptomic information that, for the first time, capture and meaningfully explain a sizable percentage of medically known comorbidities in a significant manner. This supports the idea that disease comorbidities have a strong molecular 
476 component that is better captured with gene expression profiles than with other molecular sources.

477 Actually, differential gene expression profiles portray the diseases' altered state in a rich manner since

478 its signal might reflect from genetic alterations to the epigenetic influence on gene expression due to

479 internal or external factors such as treatments, contaminants or lifestyle.

480 This study shed light into the biological processes underlying known disease comorbidities, leading to

481 a better understanding of the molecular profile and etiology of diseases and their comorbidity 482 relationships. Importantly, although we showed that many of these mechanisms have already been

483 validated experimentally, our efforts propose numerous key genes and pathways that are still to be 484 explored. Thus, we focused our discussion on some examples and provided the molecular 485 characterization of all the diseases and meta-patients at the different levels of granularity (genes and 486 pathways) within a framework that allows for the comparison of the molecular profiles for direct and inverse comorbidities in a detailed and user-friendly manner (http://diseaseperception.bsc.es/rgenexcom/).

Finally, our study stresses the need to integrate the study of disease comorbidities and their underlying molecular similarities within a personalized medicine scope, with the aim to capture those disease interactions that might depend on the disease subtype or other patient-specific factors. This would allow us, not only to better understand the putative secondary conditions of specific patients, but to better characterize the underlying molecular processes that might explain those relationships.

\section{Methods}

\section{Gene Expression Analysis}

497 Uniformly processed RNA-seq gene counts were downloaded from the GREIN platform ${ }^{13}$ for 72 human 498 diseases analyzed by 107 studies, including a total number of 4.267 samples.

499 An RNA-seq pipeline destined to the parallel processing of a collection of RNA-seq studies for a given 500 set of diseases was developed (Supplementary Fig. 12). First, samples with a percentage of aligned 501 reads to the genome lower than 70\% were removed, as well as studies with less than 3 cases (from now 502 on patients) and control samples meeting the mentioned requirement. Secondly, and in order to perform 503 the analysis at the disease level, gene counts and metadata for each disease were integrated (only studies 504 with the disease, tissue and disease state information were considered). We performed quality controls 505 using the edgeR pipeline ${ }^{58}$ and we applied within-sample normalization by considering the logarithm 506 of the counts-per-million $(\log 2 \mathrm{CPM})$. Afterwards, we filtered out lowly expressed genes (those with 
507

508

509

510

511

512

513

514

515

516

517

518

519

520

521

522

523

524

525

526

527

528

529

530

531

532

533

534

535

536

537

538

less than $1 \log 2 \mathrm{CPM}$ in more than $20 \%$ of the samples) and we applied between-sample normalization using the trimmed mean of M values (TMM) method ${ }^{59}$. After performing batch effect identification, we used the limma pipeline ${ }^{60}$ for differential expression analysis. Specifically, we built a model considering sample type (case vs. control) as our outcome of interest and adjusting for the study effect, as it is the most descriptive independent variable (tissue, platform and others depend on the study of origin). Genes with an FDR $<=0.05$ were considered significantly differentially expressed genes (sDEGs). Moreover, we used Combat ${ }^{61}$ and QR Decomposition ${ }^{60}$ batch effect removal methods to check the clustering of the samples with t-Distributed Stochastic Neighbor embedding (tSNEs) ${ }^{62}$.

\section{Functional enrichment analysis}

In order to better characterize the molecular processes underlying the analyzed diseases, we performed Gene Set Enrichment Analyses (GSEA) ${ }^{14}$ on the ranked lists of genes based on the differential expression -log Fold Change (logFC)- results using annotations from Reactome ${ }^{15}$, Kyoto Encyclopedia of Genes and Genomes ${ }^{63}$ and Gene Ontology ${ }^{64}$. We considered gene sets and pathways with an FDR $<=0.05$ to be significant.

To facilitate the interpretation of the molecular processes altered in diseases and potentially involved in disease comorbidity relationships, we selected the Reactome pathways ${ }^{15}$ significantly enriched in each disease and applied Ward2 algorithm ${ }^{38}$ to cluster diseases based on the Euclidean distance of their binarized Normalized Effect Sizes (1s and -1s for up- and down-regulated pathways) (Fig. 1).

\section{Disease similarity network}

To define disease-disease similarities we computed, for each disease pair, the Spearman's correlation between the $\operatorname{logFC}$ values of the genes in the union of their sDEGs. We kept the interactions between different diseases that were significant after correcting for multiple testing (FDR $<=0.05$ ). The resulting disease similarity network (DSN) contains positive and negative interactions (significantly positive and negative correlations respectively).

Then, we evaluated the overlap of the obtained positive interactions extracted from diseases' gene expression similarities with the ones described by Hidalgo et al. ${ }^{2}$ (based on medical records). To do so, we transformed our disease names into the International Code of Diseases, version 9 (ICD9 codes). Since some diseases share the same three-digits ICD9 code (e.g. muscular dystrophy, myotonic dystrophy and facioscapulohumeral dystrophy share the code 359 - muscular dystrophies and other myopathies), we grouped their samples together and ran the whole analysis (gene expression analysis and disease-disease network building) on them, generating an ICD9 similarity network. Next, we computed the overlap as the percentage of interactions of the epidemiological network -entailing 
common diseases- captured by the ICD9 DSN's positive and negative interactions independently. To

540 show the enrichment of our network in epidemiological interactions, we also computed the overlap in

541 the opposite direction. That is, the percentage of interactions in the ICD9 DSN contained in the

542 epidemiological network. We assessed the significance of the overlaps by shuffling the interactions

543 while preserving the degree distribution. We also computed the overlaps directly from the DSN

544 (Supplementary Notes, Supplementary Table 11).

545 Finally, we compared our overlap with the one obtained with other disease-disease networks based on 546 molecular data. We downloaded networks that link diseases based on the similarities of their 547 microbiome $^{10}$ and miRNAs ${ }^{9}$ and we generated a disease-disease network based on protein-protein 548 interactions (PPIs) by selecting the disease pairs that present a significant overlap of their network 549 modules as described by Menche et al. ${ }^{6}$. Next, we computed their overlap with the epidemiological 550 network over their common set of diseases and over the common set that is contained in our disease set. 551 Finally, we compared the ICD9-level DSN with the networks that significantly overlap the 552 epidemiology (the network based on PPIs and the microarrays' disease molecular similarity network by 553 Sánchez-Valle et al. ${ }^{8}$.

\section{Meta-patients generation}

555 We stratified diseases into subgroups of patients with similar expression profiles (meta-patients) by 556 applying clustering algorithms to the normalized and batch effect corrected gene expression matrix. Both PAM (k-medoids) ${ }^{65}$ and Ward2 ${ }^{38}$ algorithms were applied independently (Supplementary Fig. 12).

558 In the k-medoids approach, we calculated pairwise distances as 1 - the Spearman's correlation. To obtain the diseases' meta-patients, first we obtained the optimal number of clusters for each disease by running k-medoids for a cluster number between 2 and 15 . After that, the cluster number with the highest average Silhouette value was used to obtain the final meta-patients ${ }^{66}$.

562 To evaluate our approach, we selected breast cancer, a disease with known molecular subtypes and for 563 which we have two independent studies. We compared our two independently obtained clusters with 564 the defined disease subtypes (Supplementary Table 9).

\section{Stratified Similarity Network}

566 To analyze the disease subtype-associated comorbidities, we built the Stratified Similarity Network 567 (SSN) connecting meta-patients and diseases based on the pairwise Spearman's correlation of the union 568 of their sDEGs. First, meta-patient's gene expression analysis was performed using the same approach described for the diseases, where all the samples corresponding to a given meta-patient were compared 
with all the controls for the disease. Then, the SSN was built following the same methodology described

571 for the DSN by treating meta-patients and diseases equally as phenotypes. To assess if the meta-patients

572 increase the detection power significantly, we generated 1000 random meta-patients for each disease

573 by shuffling the cases while maintaining the meta-patients' number and size. Next, we obtained 1000

574 SSNs and evaluated if the number of positive and negative interactions in the SSN could be observed

575 by chance. To evaluate if meta-patients capture epidemiologically known associations with diseases,

576 we selected the positive interactions between meta-patients and diseases, transformed them into ICD9

577 codes and computed their overlap with the epidemiological network from Hidalgo et $a l^{2}$, as described

578 for the DSN. This is comparable to the available epidemiological network, that comprises interactions

579 at the disease level by evaluating if a group of patients from a given disease is at a higher risk of

580 developing a specific secondary condition.

\section{Web application}

582 To facilitate the visualization and exploration of the generated networks, we implemented a web 583 application that displays the DSN and SSN in a dynamic manner ${ }^{67}$. The user can filter the networks by

584 the type of interactions (positive or negative) and by selecting a minimum and maximum threshold for

585 the edge's weight. Community detection algorithms (greedy modularity optimization ${ }^{68}$ or random 586 walks ${ }^{69}$ can be applied to the filtered network and interactions involving specific nodes can be filtered 587 and highlighted. Furthermore, the molecular mechanisms behind diseases and disease interactions can

588 be easily inspected and compared.

589

590 Data Availability

591 The code of the experiments is available at https://github.com/beatrizurda/Urda-Garcia_et_al_2021 and 592 the code of the web application can be found at https:/github.com/bsc-life/rgenexcom. The data is

593 publicly available (Supplementary Data 1); the raw data can be downloaded from GEO 594 (https://www.ncbi.nlm.nih.gov/geo/) and the counts can be downloaded from the GREIN platform 595 (http://www.ilincs.org/apps/grein/).

596

\section{Acknowledgments}

598 This work has been supported by the Ph.D. Fellowship (PRE2019-090454) and funded by the Spanish 599 Ministry of Economics and Competitiveness (RTI2018-096653-B-I00). J.S.-V. was supported by the 600 PhD fellowship BES-2016-077403. 
601 We thank Vera Pancaldi from the Centre de Recherches en Cancérologie de Toulouse, INSERM and

602 Barcelona Supercomputing Center (BSC); Anaïs Baudot from the INSERM, Marseille Medical

603 Genetics and BSC, Jose Luis Portero Navío from Novo Nordisk; Emre Guney from Hospital del Mar

604 Medical Research Institute and Universitat Pompeu Fabra, and Marta Mele from BSC for their helpful 605 critical comments on the work.

606

\section{Contributions}

608 B.U.-G, J.S.-V., R.L. and A.V. designed all the experiments. B.U.-G. performed all the experiments 609 and developed the web application. All the authors wrote the manuscript and discussed the obtained 610 results.

611 Conflict of Interest: none declared.

612

\section{References}

614 1. Valderas, J. M., Starfield, B., Sibbald, B., Salisbury, C. \& Roland, M. Defining comorbidity: 615 Implications for understanding health and health services. Ann. Fam. Med. 7, 357-363 (2009).

616 2. Hidalgo, C. A., Blumm, N., Barabási, A. L. \& Christakis, N. A. A Dynamic Network 617 Approach for the Study of Human Phenotypes. PLoS Comput. Biol. 5, e1000353 (2009).

618 3. Jiang, Y., Ma, S., Shia, B. C. \& Lee, T. S. An epidemiological human disease network derived 619 from disease Co-occurrence in Taiwan. Sci. Rep. 8, 4557 (2018).

620 4. Jensen, A. B. et al. Temporal disease trajectories condensed from population-wide registry 621 data covering 6.2 million patients. Nat. Commun. 5, 4022 (2014).

622 5. Goh, K. Il et al. The human disease network. Proc. Natl. Acad. Sci. U. S. A. 104, 8685-8690 623 (2007).

624 6. Menche, J. et al. Uncovering disease-disease relationships through the incomplete interactome. $625 \quad$ Science. 347, $841(2015)$.

626 7. Ibáñez, K., Boullosa, C., Tabarés-Seisdedos, R., Baudot, A. \& Valencia, A. Molecular 627 Evidence for the Inverse Comorbidity between Central Nervous System Disorders and Cancers Detected by Transcriptomic Meta-analyses. PLoS Genet. 10, 4022 (2014).

629 8. Sánchez-Valle, J. et al. Interpreting molecular similarity between patients as a determinant of 

disease comorbidity relationships. Nat. Commun. 11, 2854 (2020).

631 9. Lu, M. et al. An analysis of human microRNA and disease associations. PLoS One 3, e3420

632 (2008).

633

10. Ma, W. et al. An analysis of human microbe-disease associations. Brief. Bioinform. 18, 85-97

634 (2017).

635

11. Hrdlickova, R., Toloue, M. \& Tian, B. RNA-Seq methods for transcriptome analysis. Wiley Interdiscip. Rev. RNA 8, (2017).

637

12. Costa-Silva, J., Domingues, D. \& Lopes, F. M. RNA-Seq differential expression analysis: An

638 extended review and a software tool. PLoS One 12, e0190152 (2017).

639

13. Mahi, N. Al, Najafabadi, M. F., Pilarczyk, M., Kouril, M. \& Medvedovic, M. GREIN: An

640 Interactive Web Platform for Re-analyzing GEO RNA-seq Data. Sci. Rep. 9, 7580 (2019).

641

14. Subramanian, A. et al. Gene set enrichment analysis: A knowledge-based approach for

642

643 interpreting genome-wide expression profiles. Proc. Natl. Acad. Sci. U. S. A. 102, $15545-$ 15550 (2005).

644

15. Fabregat, A. et al. The reactome pathway knowledgebase. Nucleic Acids Res. 44, D481-D487

645 (2016).

646

16. Theocharis, A. D., Manou, D. \& Karamanos, N. K. The extracellular matrix as a multitasking

647 player in disease. FEBS J. 286, 2830-2869 (2019).

648 17. Barros, C. S., Franco, S. J. \& Müller, U. Extracellular Matrix: Functions in the nervous system. Cold Spring Harb. Perspect. Biol. 3, 1-24 (2011).

650

18. Sausville, E. A., Elsayed, Y., Monga, M. \& Kim, G. Signal Transduction-Directed Cancer Treatments. Annu. Rev. Pharmacol. Toxicol. 43, 199-231 (2003).

652

19. M. Candeias, S. \& S. Gaipl, U. The Immune System in Cancer Prevention, Development and Therapy. Anticancer. Agents Med. Chem. 16, 101-107 (2015).

20. Cooper, G. M. \& Hausman, R. E. The Development and Causes of Cancer. The Cell: A Molecular Approach (Sinauer Associates, 2007).

21. Petrey, A. C. \& De La Motte, C. A. The extracellular matrix in IBD: A dynamic mediator of inflammation. Curr. Opin. Gastroenterol. 33, 234-238 (2017). 
22. Shahin, M. et al. Remodeling of extracellular matrix in gastric ulceration. Microsc. Res. Tech. 53, 396-408 (2001).

23. Stankevicius, V. et al. Extracellular matrix-dependent pathways in colorectal cancer cell lines reveal potential targets for anticancer therapies. Anticancer Res. 36, 4559-4567 (2016).

24. Hoffmann, C., Sabranski, M. \& Esser, S. HIV-Associated Kaposi’s Sarcoma. Oncol. Res. Treat. 40, 94-98 (2017).

25. Rodríguez-Peláez, M. et al. Kaposi's sarcoma: An opportunistic infection by human herpesvirus-8 in ulcerative colitis. J. Crohn's Colitis 4, 586-590 (2010).

26. Kilincalp, S., Akinci, H., Hamamci, M., Coşkun, Y. \& Yüksel, I. Kaposi’s sarcoma developing in a HIV-negative Crohn's disease patient shortly after azathioprine and corticosteroid treatment. J. Crohn's Colitis 8, 558-559 (2014).

27. JA, D. Inverse association between cancer and neurodegenerative disease: review of the epidemiologic and biological evidence. Biogerontology 15, 547-557 (2014).

28. McNulty, P. et al. Reduced Cancer Incidence in Huntington's Disease: Analysis in the Registry Study. J. Huntingtons. Dis. 7, 209-222 (2018).

29. Rath, O. \& Kozielski, F. Kinesins and cancer. Nat. Rev. Cancer 12, 527-539 (2012).

30. DeBerg, H. A. et al. Motor domain phosphorylation modulates kinesin-1 transport. J. Biol. Chem. 288, 32612-32621 (2013).

31. Soulet, D. \& Cicchetti, F. The role of immunity in Huntington's disease. Mol. Psychiatry 16, 889-902 (2011).

32. Gonzalez, H., Hagerling, C. \& Werb, Z. Roles of the immune system in cancer: From tumor initiation to metastatic progression. Genes Dev. 32, 1267-1284 (2018).

33. Lu, X. Impact of IL-12 in Cancer. Curr. Cancer Drug Targets 17, 682-697 (2017).

34. Logan, R. W. \& McClung, C. A. Rhythms of life: circadian disruption and brain disorders across the lifespan. Nat. Rev. Neurosci. 20, 49-65 (2019).

35. Hood, S. \& Amir, S. Neurodegeneration and the circadian clock. Front. Aging Neurosci. 9, 170 (2017).

36. Barandas, R., Landgraf, D., McCarthy, M. J. \& Welsh, D. K. Circadian Clocks as Modulators 
37. Park, H. S. \& Jun, C. H. A simple and fast algorithm for K-medoids clustering. Expert Syst. Appl. 36, 3336-3341 (2009).

689

38. Murtagh, F. \& Legendre, P. Ward's Hierarchical Clustering Method: Clustering Criterion and Agglomerative Algorithm. 31, 274-295, (2014).

39. Hu, J. X., Thomas, C. E. \& Brunak, S. Network biology concepts in complex disease comorbidities. Nat. Rev. Genet. 17, 615-629 (2016).

40. Nebbia, M., Yassin, N. A. \& Spinelli, A. Colorectal Cancer in Inflammatory Bowel Disease. Clin. Colon Rectal Surg. 33, 305-317 (2020).

41. Bonsignore, M. R. \& Steiropoulos, P. Metabolic syndrome. ERS Monograph vol. 2015 (StatPearls Publishing, 2015).

42. Zhou, X. et al. A Systems Approach to Refine Disease Taxonomy by Integrating Phenotypic and Molecular Networks. EBioMedicine 31, 79-91 (2018).

43. Kibune-Nagasako, C., Garcia-Montes, C., Silva-Lorena, S. L. \& Aparecida-Mesquita, M. Irritable bowel syndrome subtypes: Clinical and psychological features, body mass index and comorbidities. Rev. Esp. Enfermedades Dig. 108, 59-64 (2016).

44. Tsai, F. J., Tseng, W. L., Yang, L. K. \& Gau, S. S. F. Psychiatric comorbid patterns in adults with attention-deficit hyperactivity disorder: Treatment effect and subtypes. PLoS One 14, e0211873 (2019).

45. Witthauer, C. et al. Associations of specific phobia and its subtypes with physical diseases: An adult community study. BMC Psychiatry 16, 16:155 (2016).

46. Fairthorne, J., Hammond, G., Bourke, J., Jacoby, P. \& Leonard, H. Early mortality and primary causes of death in mothers of children with intellectual disability or Autism spectrum disorder: A retrospective cohort study. PLoS One 9, e113430 (2014).

47. Mouridsen, S. E., Rich, B. \& Isager, T. Risk of cancer in adult people diagnosed with infantile autism in childhood: A longitudinal case control study based on hospital discharge diagnoses.

713 48. Forés-Martos, J. et al. Transcriptomic metaanalyses of autistic brains reveals shared gene 
expression and biological pathway abnormalities with cancer. Mol. Autism 10, 17 (2019).

715

716

717

718

719

720

721

722

723

724

725

726

727

728

729

730

731

732

733

734

735

736

737

738

739

740

741

49. Kao, H.-T., Buka, S. L., Kelsey, K. T., Gruber, D. F. \& Porton, B. The correlation between rates of cancer and autism: an exploratory ecological investigation. PLoS One 5, e9372-e9372 (2010).

50. BarChana, M. et al. Enhanced cancer risk among patients with bipolar disorder. J. Affect. Disord. 108, 43-48 (2008).

51. O’Malley, P. W., Mulla, Z. D. \& Nesic, O. Multiple sclerosis and breast cancer. J. Neurol. Sci. 356, 137-141 (2015).

52. Etemadifar, M. et al. Cancer risk among patients with multiple sclerosis: A cohort study in Isfahan, Iran. Casp. J. Intern. Med. 8, 172-177 (2017).

53. Tabarés-Seisdedos, R. et al. No paradox, no progress: Inverse cancer comorbidity in people with other complex diseases. Lancet Oncol. 12, 604-608 (2011).

54. Sung, Y. F. et al. Reduced Risk of Parkinson Disease in Patients With Rheumatoid Arthritis: A Nationwide Population-Based Study. Mayo Clin. Proc. 91, 1346-1353 (2016).

55. Westergaard, D., Moseley, P., Sørup, F. K. H., Baldi, P. \& Brunak, S. Population-wide analysis of differences in disease progression patterns in men and women. Nat. Commun. 10, 666 (2019).

56. B, B., J, P. \& M, S. Computational solutions for omics data. Nat. Rev. Genet. 14, 333-346 (2013).

57. Lee, Y. suk et al. A Computational Framework for Genome-wide Characterization of the Human Disease Landscape. Cell Syst. 8, 152-162.e6 (2019).

58. Robinson, M. D., McCarthy, D. J. \& Smyth, G. K. edgeR: A Bioconductor package for differential expression analysis of digital gene expression data. Bioinformatics 26, 139-140 (2009).

59. Robinson, M. D. \& Oshlack, A. A scaling normalization method for differential expression analysis of RNA-seq data. Genome Biol. 11, 184-99 (2010).

60. Ritchie, M. E. et al. Limma powers differential expression analyses for RNA-sequencing and microarray studies. Nucleic Acids Res. 43, e47 (2015). 
742 61. Johnson, W. E., Li, C. \& Rabinovic, A. Adjusting batch effects in microarray expression data $743 \quad$ using empirical Bayes methods. Biostatistics 8, 118-127 (2007).

744 62. Van Der Maaten, L. \& Hinton, G. Visualizing data using t-SNE. J. Mach. Learn. Res. 9, 2579$745 \quad 2625(2008)$.

746 63. Kanehisa, M. \& Goto, S. KEGG: kyoto encyclopedia of genes and genomes. Nucleic Acids Res. 28, 27-30 (2000).

748 64. Ashburner, M. et al. Gene ontology: Tool for the unification of biology. Nat. Genet. 25, 25-29 (2000).

750 65. Maechler, M. et al. Package 'cluster': Cluster Analysis Basics and Extensions. R topics Documented 79 (2021).

66. Rousseeuw, P. J. Silhouettes: A graphical aid to the interpretation and validation of cluster analysis. J. Comput. Appl. Math. 20, 53-65 (1987).

754 67. Chang, W. et al. shiny: Web Application Framework for R. R package (2021).

68. Pons, P. \& Latapy, M. Computing Communities in Large Networks Using Random Walks. $J$. Graph Algorithms Appl. 10, 191-218 (2006).

757 69. Clauset, A., Newman, M. E. J. \& Moore, C. Finding community structure in very large networks. Phys. Rev. E - Stat. Physics, Plasmas, Fluids, Relat. Interdiscip. Top. 70, 6 (2004). 